\title{
EFFECT OF TWO DIFFERENT HANDGRIP POSITIONS ON ELBOW PEAK TORQUE VALUES
}

\author{
Leon LATEGAN* \& P.E. (Ernst) KRÜGER** \\ * Department of Sport and Movement Studies, University of Johannesburg, Johannesburg, \\ Republic of South Africa \\ **Department of Biokinetics, Sport and Leisure Sciences, University of Pretoria, Pretoria, \\ Republic of South Africa
}

\begin{abstract}
The purpose of this study was to determine the effect of using two different handgrip positions on the peak torque values of the elbow during flexion and extension and to establish normative concentric isokinetic values for elbow flexion, extension and the flexion/extension ratio at $60 \%$ s. Four hundred and thirty-eight $(n=438)$ healthy South African males, between the ages of 16 and 29 years were used as subjects. A Cybex 340 isokinetic dynamometer was used to determine concentric peak torque of the non-dominant elbow's flexion and extension, using two different handgrip positions. Using the anatomical zero (AZ) handgrip (Group A) resulted in a mean elbow flexion of $78 \%$ of body mass (BM) and an elbow extension torque of $67 \%$ BM, compared to $69 \%$ BM and $86 \%$ BM, respectively when using the $90^{\circ}$ pronated handgrip (Group $B$ ). The flexion/extension ratio was $119 \%$ for the AZ handgrip and $82 \%$ for the $90^{\circ}$ pronated handgrip position. Thus, significant differences were demonstrated at a 99.9\% confidence level ( $p \leq 0.001)$ between the two groups, when using these two handgrip positions. Additionally, normative absolute peak torque values (Nm) and weight-normalised normative torque values (\% BM) for concentric isokinetic elbow flexion and extension were established. This may provide clinicians with applicable normative values for rehabilitation and sport science purposes.
\end{abstract}

Key words: Isokinetics; Elbow; Strength; Torque.

\section{INTRODUCTION}

The increased use of isokinetic evaluation and isokinetic exercise has created a need for normative values, both for rehabilitation and sport science purposes. This is especially true for joints that have not been studied to a great extent, i.e. the wrist, elbow and hip joints. There exist very limited isokinetic data on the elbow joint compared to that of the knee joint. There is also a need for normative values that take age and body weight into account to make predictions of peak torque and agonist-antagonist ratio's in a variety of populations (Dvir, 2004).

Isokinetic norms may be used for setting goals during rehabilitation and for pre-participation screening of elite athletes (for muscle weakness or imbalances). However, when dealing with athletes, isokinetic testing has its limitations. During a tennis serve for example, elbow extension may reach velocities between $982 \%$ s and 1700\% (Dillman, 1991; Kibler, 1994). Furthermore, Osternig (1975) demonstrated that the isokinetic load range decreases from $92 \%$ to $16 \%$ at isokinetic speeds of $50 \%$ s to $400 \%$ s. This implies that high-speed isokinetics may 
have such a small load range that it may become ineffective as far as an "isokinetic" training/testing method is concerned. Taylor et al. (1991) also reported increased errors in isokinetic testing with increased velocity (from $60 \%$ s to $450 \%$ s). The above limitations should caution researchers and clinicians not to utilize extremely fast isokinetic speeds $\left(>240^{\circ} / \mathrm{s}\right)$ isokinetic speeds when testing the elbow joint (Kibler, 1994).

When evaluating the elbow's flexors and extensors, there are two main set-up procedures that may influence test results. The first involves an anatomical zero handgrip position (AZ-pos) with the forearm in full supination. The second method has a handgrip position with the forearm pronated to $90^{\circ}$ from full supination $\left(90^{\circ}\right.$-pos). This position is normally favoured by clinicians when testing the elbow joint (Dvir, 2004). By changing the position of the handgrip, the angles of pull change. By performing elbow flexion with a fully supinated handgrip, the biceps brachii are at an optimal angle of pull and elbow flexion torque is enhanced (Lategan, 2002). When the forearm is pronated, elbow flexion peak torque is reduced since the biceps brachii are now disadvantaged with regards to angle of pull. Although the brachioradialis appears to have a slightly stronger action in the pronated position this muscle is contributing less to the total flexion strength. The position of the forearm does not affect the contraction strength of the brachialis in elbow flexion (Hamilton \& Luttgens, 2002; Kendall et al., 2005). Extension of the elbow is also affected by a change in handgrip-position. By pronating the forearm, elbow extension force is increased, possibly due to a favourable angle of pull in the lateral head of the triceps brachii (Hamilton \& Luttgens, 2002).

According to previous research, the concentric elbow flexion peak torque (PT) divided by elbow extension PT (expressed as a percentage) at slower isokinetic speeds $(30 \% \mathrm{~s}$ and $60 \% \mathrm{~s})$ should be between 90\% and 100\% (Perrin, 1993; Heyward, 1997). Knapik and Ramos (1980) investigated concentric isokinetic elbow flexion and extension peak torque (at 30, 90 and $180 \%$ s) in 352 male infantry soldiers using the dominant side only and utilizing the AZhandgrip position. At $30 \%$, they reported an elbow flexion peak torque of $50 \mathrm{Nm}(67 \% \mathrm{BW})$, an extension peak torque value of $44 \mathrm{Nm}(59 \% \mathrm{BW})$ and a flexion/extension ratio of $114 \%$. At $90 \%$ s Knapik and Ramos (1980) reported elbow flexion peak torque values of $36 \mathrm{Nm}(48 \%$ BW) and $29 \mathrm{Nm}(39 \% \mathrm{BW})$. The flexion/extension ratio was $109 \%$, which is in accordance with the range suggested above. In addition, Ellenbecker and Mattalino (1997) reported an elbow flexion/extension peak torque ratio of $103 \%$ for professional baseball pitchers' dominant side and $101 \%$ for the non-dominant side, using the AZ-pos. In elite male junior tennis players, an elbow flexion-extension ratio of $97 \%$ and $108 \%$ was reported for the dominant and non-dominant side, respectively at $90 \%$ s (Ellenbecker \& Roetert, 2000).

Although the results from Knapik and Ramos (1980) provide useful data at angular velocities of $30 \%$ s, $90 \%$ and $180^{\circ} \%$, normative isokinetic torque values for the elbow at $60 \% \mathrm{~s}(\mathrm{most}$ standardized isokinetic protocols include $60 \%$ s for slow speed testing) are largely absent from the available literature (Chan \& Maffulli, 1996; Wrigley \& Strauss, 2000; Dvir, 2004); this despite $60 \%$ s being the most popular and commonly used of slow angular testing velocities in the majority of joints (Wrigley \& Strauss, 2000; Mandalidis \& O'Brien, 2001; Dvir, 2004; Olmo et al., 2006). There is also very little information regarding norms when using the $60^{\circ}$ pronated handgrip position (Perrin, 1993). 
Two research studies did however, report on elbow torque values using the $90^{\circ}$ pronated grip position in men, but due to the limited number of subjects one should be cautious to adopt their results as normative for the elbow (Frontera et al., 1993; Mandalidis \& O’Brien, 2001).

Thus, the purpose of this study was to determine the effect of using two different handgrip positions on the peak torque values of the elbow during flexion and extension and to establish normative concentric isokinetic values for elbow flexion, extension and the flexion/extension ratio at a velocity of $60^{\circ} / \mathrm{s}$.

\section{METHODS}

The research design used was an experimental, quantitative, and comparative analysis.

\section{Instrumentation}

A computerized Cybex 340 isokinetic dynamometer (Cybex, A division of Lumex, Inc., 2100 Smithtown Avenue, Ronkonkoma, New York) was used to measure muscular torque. A Harpenden skinfold calliper (Harpenden John Bull, British Indicators Ltd., England) was used to determine percentage body fat.

\section{Subjects}

Four hundred and thirty-eight (438) South African males between the ages of 16 and 29 years $(19.07 \pm 1.91)$ were used as subjects and the testing took place over a 3-year period. The subjects were divided into two groups according to the calendar year in which they were tested. Group A, who was tested in the first calendar year, used an anatomical zero (AZ) handgrip position (i.e. forearm supinated fully) and Group B (second and third calendar year), made use of a $90^{\circ}$-pronated handgrip. The non-dominant side was used for testing due to the enormous size of the sample (438 subjects) and to prevent using the elevated peak torque values from the subjects' dominant side (it is well-known that the dominant side may have peak torque values of between 5\% and 20\% higher than the non-dominant side) (Perrin, 1993). Group A consisted of 199 subjects and Group B of 239 subjects. The subjects' activity levels ranged from inactive to athletes and were thus heterogeneous with regards to activity level.

A medical doctor examined every subject before the physical testing commenced, in order to exclude orthopaedic injury or any other medical condition deemed contra-indicative to the subject participation in physical activity (e.g. diabetes mellitus, recent myocardial infarction, hypertension or an acute infection) (ACSM, 2006; Heyward, 1997). After a thorough explanation of the testing procedure, every subject completed and signed an informed consent to participate voluntarily in the study.

\section{Anthropometrical data}

Personal and anthropometrical data, including age, body mass, height and skinfolds were obtained before the testing commenced. The MOGAP method was used to calculate percentage body fat (Carter et al., 1982). 


\section{Warm-up}

Warm-up consisted of light jogging for five minutes, static stretching of the involved muscle groups, as well as four isokinetic repetitions. The first two isokinetic contractions were performed at a perceived effort of $50 \%$, the next repetition at $75 \%$, and the last repetition at $100 \%$ of perceived maximum voluntary contraction (MVC).

\section{Isokinetic testing protocol}

Each subject was positioned and stabilized according to the Cybex 340 User Manual. A damp setting value of 2 was used during all the testing and the Cybex was calibrated after every fourth session of testing. No correction was made for the effects of gravity. The non-dominant side was used throughout and only the concentric contraction mode was utilized.

The two handgrip positions utilized were:

- $\quad$ anatomical zero (AZ) position, e.g. full forearm supination (Group A);

- $90^{\circ}$ pronated position $\left(90^{\circ}\right.$ pos.), e.g. forearm was pronated $90^{\circ}$ (Group B).

During isokinetic testing, the active ROM ranged from full elbow extension ( $0^{\circ}$ flexion) to the position of maximal elbow flexion. The total ROM used was thus approximately $165^{\circ}$. A mechanical ROM stop was used to prevent hyperextension of the elbow.

Following the four warm-up repetitions, five reciprocal maximum concentric elbow flexion and extension repetitions were performed at an angular velocity of $60^{\circ} / \mathrm{sec}$. Subjects were all verbally motivated to provide maximum effort during all five repetitions. If subjects expressed the view that they were not able to achieve their maximum effort due to unfamiliarity with the testing protocol, they were allowed to rest for five minutes before repeating the test. Subjects who experienced discomfort during any of the testing were excused and excluded from the study.

\section{Interpretation of isokinetic data}

The peak torque of each subject was determined by selecting the largest peak torque obtained in five repetitions. Absolute peak torque was expressed in Newton metres $(\mathrm{Nm})$ and relative peak torque was expressed as the percentage torque per kilogram body mass (\%). Elbow flexion/extension (\%) ratios were calculated for both groups (A \& B). None of the results were corrected for the effect of gravity.

\section{Statistical analysis}

The group average, minimum, maximum and standard deviation were calculated for each movement pattern using descriptive statistical methods. The independent student t-Test (as suggested by our University's statistical consultation services - STATCON) was conducted to establish whether there was a significant difference between the two groups. A confidence level of $99.9 \%(\mathrm{p}<0.001)$ was used due to the large sample. 


\section{RESULTS AND DISCUSSION}

\section{Kinanthropometrical data}

The anthropometrical data of Group A and B are presented in Table 1. Group A and Group B were homogeneous regarding kinanthropometrical profiles (age, body mass, percentage body fat, and lean body mass), at a $99.9 \%$ confidence level ( $\mathrm{p}>0.001)$. Thus, we expected the two groups to also display homogeneous torque values for the elbow joint, especially since they had similar values for lean body mass $(65.4 \mathrm{~kg} \pm 6.78 \mathrm{vs} .63 .4 \mathrm{~kg} \pm 6.90)$.

\section{TABLE 1: SUBJECT CHARACTERISTICS}

\begin{tabular}{|l|l|l|l|l|l|}
\hline & \multicolumn{2}{|l|}{ Group A (AZ-pos.) } & \multicolumn{2}{l|}{ Group B (90-pos.) } & $\begin{array}{l}\text { ANOVA } \\
\text { between } \\
\text { group means }\end{array}$ \\
\hline & Mean & STD & Mean & STD & p-value \\
\hline & & & & & \\
\hline Age & 19.32 & 1.80 & 18.88 & 1.98 & $0.0152 \mathrm{NS}$ \\
\hline Body mass (kg) & 72.73 & 8.58 & 70.65 & 9.34 & $0.0154 \mathrm{NS}$ \\
\hline Percentage body fat & 9.96 & 2.88 & 9.98 & 3.46 & $0.6836 \mathrm{NS}$ \\
\hline Lean body mass (kg) & 65.41 & 6.78 & 63.39 & 6.90 & $0.0022 \mathrm{NS}$ \\
\hline
\end{tabular}

NS: No significant difference between Group A and Group B at a $99.9 \%$ confidence level ( $>0.001$ ) was found; the two groups were thus homogenous.

\section{Isokinetic peak torque values}

There was a significant difference of $14.2 \%(\mathrm{p}<0.001)$ in elbow flexion peak torque between the two groups. Group A reported a mean elbow flexion of $56.5 \mathrm{Nm}$, compared to $48.5 \mathrm{Nm}$ for Group B (Table 2). When expressed as percentage of body mass, the difference between Group A $(77.7 \%)$ and group B $(69.1 \%)$ was found to be statistically significant $(p<0.001)$. There was also a significant difference of $20.2 \%(p<0.001)$ in elbow extension torque between the two groups. Group A reported a mean elbow extension of $48.4 \mathrm{Nm}$, compared to $60.6 \mathrm{Nm}$ for Group B (Table 2). When these values were expressed as a percentage of body mass, the difference between Group A (66.5\%) and Group B (86.0\%) was found to be significantly different $(\mathrm{p}<0.001)$. The elbow flexion/extension ratio for Group A $(119 \%)$, compared to Group B (81.7\%) thus differed by $37.3 \%$ (Table 2 and Figure 2 ).

Thus, Group A (AZ pos.) and Group B ( $90^{\circ}$ pos.) demonstrated significant inter-group differences at a $99.9 \%$ confidence level $(\mathrm{p}<0.001)$ for both elbow flexion and extension peak torque and for peak torque as a percentage of body mass. There was also a significant difference in elbow flexion/extension ratio between the two groups (Table 2). Since these two groups were homogeneous with regard to their kinanthropometrical profiles, these differences in isokinetic elbow flexion and extension peak torque may be attributed to the use of the two 
different handgrip positions (AZ pos. vs. $90^{\circ}$ pos.). These findings support the previous work done by researchers who stated that by changing the forearm position and angles of pull of the involved muscles, one can also change the muscles' MVC force (Hamilton \& Luttgens, 2002; Kendall et al., 2005). With the AZ handgrip position (Group A), the elbow flexors (e.g. brachioradialis) are at a biomechanical advantage during elbow flexion compared to the $90^{\circ}$ pronated handgrip position (Group B). However, the $90^{\circ}$ pronated handgrip position (Group B) provided the elbow extensors (triceps brachii) with an advantage during elbow extension compared to the AZ handgrip position (Group A) (Kendall et al., 2005).

\section{TABLE 2: ELBOW FLEXION AND EXTENSION PEAK TORQUE AT 60\%/S}

\begin{tabular}{|l|l|r|l|r|l|}
\hline & \multicolumn{2}{l|}{ Group A (AZ pos.) } & \multicolumn{2}{l|}{$\begin{array}{l}\text { Group B }\left(90^{\circ}\right. \\
\text { pos.) }\end{array}$} & $\begin{array}{l}\text { ANOVA } \\
\text { between group } \\
\text { means }\end{array}$ \\
\hline Movement pattern & Mean & STD & Mean & STD & p-value \\
\hline Elbow flexion: & & & & & \\
\hline Peak torque (Nm) & 56.52 & 11.14 & 48.50 & 9.6 & $7.66777 \mathrm{E}-15^{*}$ \\
\hline Peak torque/BM (\%) & 77.71 & 12.42 & 69.10 & 10.9 & $5.45346 \mathrm{E}-14 *$ \\
\hline & & & & & \\
\hline Elbow extension: & & & & & \\
\hline Peak torque (Nm) & 48.38 & 10.85 & 60.60 & 14.2 & $1.17172 \mathrm{E}-18 *$ \\
\hline Peak torque/BM (\%) & 66.47 & 12.48 & 85.97 & 15.9 & $7.69352 \mathrm{E}-37 *$ \\
\hline & & & & & \\
\hline Elbow flexion/extension (\%) & 119.02 & 19.82 & 81.74 & 14.0 & $4.2011 \mathrm{E}-77 *$ \\
\hline
\end{tabular}

*: Significant difference between Group A and Group B at a $99.9 \%$ confidence level $(\mathrm{p}<0.001)$.

When the results of the present study are compared to those of Frontera et al. (1993), Chan and Maffulli (1996) and Mandalidis and O'Brien (2001), the following become apparent. The present study's elbow flexion peak torque $(48 \mathrm{Nm})$ when using the $90^{\circ}$ pronated handgrip position, is slightly lower compared to that of Mandalidis and O'Brien (2001) (50.8-52.1 Nm) whose subjects' average age was 30 years, fairly similar to that of Frontera et al. (1993) (45 $\mathrm{Nm}$ ), but higher than that of sedentary male subjects (18-30 years (Chan \& Maffulli, 1996).

Since the study by Mandalidis and O'Brien (2001) only focussed on elbow flexion, the results of the present study were compared with that of Frontera et al. (1993). The $90^{\circ}$ pronated handgrip position resulted in a superior elbow extension peak torque of $61 \mathrm{Nm}$ in the present study, compared to the $42 \mathrm{Nm}$, reported by Frontera et al. (1993). It must however, be mentioned that the average age of the subjects from the present study (18.88 years) was considerably lower than that of Frontera et al. (1993), e.g. 45 to 78 years. Compared to the elbow extension torque of $39 \mathrm{Nm}$ reported by Chan and Maffulli (1996), the present study's results are also higher, possibly due to differences in body mass between the South African and Hong Kong subjects. With regards to the elbow flexion/extension ratio, the present study's average ratio was $82 \%$ compared to the $91 \%$ reported by Chan and Maffulli (1996) for 
sedentary males in Hong Kong. Thus, any normative values should be used with caution when extrapolating it to another population.

In the absence of local normative values for the elbow joint, the results of the present study could be used as a normative database for young South African men at a $60 \% \mathrm{~s}$ isokinetic velocity.

Although both absolute $(\mathrm{Nm})$ and relative torque values $(\% \mathrm{BW})$ were presented, the values relative to body mass (\% BW) are normally more useful, especially when proposing normative values. They eliminate the effect of body mass on peak torque production, so that the normative values may be applicable to a larger range of subjects, irrespective of body mass.

Because the present authors evaluated only the non-dominant extremity, it may be necessary to add $5 \%$ to $20 \%$ to the absolute peak torque values when considering a subject's dominant side, when setting rehabilitation goals, especially when dealing with throwing athletes, e.g. javelin, shot-put or tennis (Perrin, 1993; Wilk et al., 1993).

\section{SUMMARY}

Changing handgrip position between $\mathrm{AZ}$ position and the $90^{\circ}$ position will significantly change the elbow's flexion and extension peak torque values as well as the elbow flexion/extension ratios. Researchers and clinicians are therefore cautioned to carefully note the handgrip position when evaluating isokinetic elbow flexion and extension, especially when making comparisons with normative values.

In the absence of another large and reliable normative database for concentric isokinetic elbow flexion and extension peak torque at $60 \% \mathrm{~s}$, the present study provides the clinician and researcher with norms for young and healthy adult males. Although the values are for South African men, other populations (with a comparable kinanthropometrical profile) may possibly display similar torque characteristics. However, much more research is needed regarding isokinetic values in the elbow joint, particularly in other age groups and in female populations.

\section{REFERENCES}

ACSM (2006). ACSM's Guidelines for exercise testing and prescription. Baltimore: Williams \& Wilkins.

CARTER, J.E.L.; AUBRY, S.P. \& SLEET, D.A. (1982). Somatotypes of Montreal Olympic athletes. In J.E.L. Carter (Ed.). Physical stature of Olympic athletes. Part 1. Montreal Olympic Games Anthropological Project (pp. 53-80). Basel: Karger.

CHAN, K.M. \& MAFFULLI, N. (1996). Principles and practice of isokinetics in sports medicine and rehabilitation. Hong Kong: Williams \& Wilkins.

DILLMAN, C.J. (1991). The upper extremity in tennis and throwing athletes. Paper presented at the United States Tennis Association National Meeting, Tuscon, AZ.

DVIR, Z. (2004). Isokinetics: muscle testing, interpretation and clinical applications. New York, NY: Churchill Livingstone.

ELLENBECKER, T.S. \& MATTALINO, A.J. (1997). The Elbow in Sport. Champaign, IL: Human Kinetics. 
ELLENBECKER, T.S. \& ROETERT, E.P. (2000). Isokinetic testing and training in tennis. In L.E. Brown (Ed.). Isokinetics in human performance (pp. 358-377). Champaign, IL: Human Kinetics.

FRONTERA, W.R.; HUGHES, V.A.; DALLAL, G.E. \& EVENS, W.J. (1993). Reliability of isokinetic muscle strength testing in 45- to 78-year old men and women. Archives of Physical Medicine and Rehabilitation, 74: 1181-1185.

HAMILTON, N. \& LUTTGENS, K. (2002). Kinesiology: scientific basis of human motion. New York, NY: McGraw-Hill.

HEYWARD, V.H. (1997). Advanced fitness assessment and exercise prescription. Champaign, IL: Human Kinetics.

KENDALL, F.P.; McCREARY, E.K.; PROVANCE, P.G.; RODGERS, M.M. \& ROMANI, W.A. (2005). Muscles testing and function: with posture and pain. Baltimore, MD: Williams \& Wilkens.

KIBLER, W.B. (1994). Clinical biomechanics of the elbow in tennis: implications for evaluation and diagnosis. Medicine and Science in Sports and Exercise, 26: 1203-1206.

KNAPIK, J.J. \& RAMOS, M.U. (1980). Isokinetic and isometric torque relationships in the human body. Archives of Physical Medicine and Rehabilitation, 61(Feb): 64-67.

LATEGAN, L. (2002). Normative isokinetic torque values for rehabilitation in South Africa. Unpublished doctoral dissertation. Pretoria: University of Pretoria.

MANDALIDIS, D.G. \& O'BRIEN, M.O. (2001). Isokinetic strength of the elbow flexors with the arm in supination and in the neutral position. Isokinetics and Exercise Science, 9: 111-118.

OLMO, J.; LOPEZ-ILLESCAS, A.; MARTIN, I.; JATO, S. \& RODRIGUEZ, L.P. (2006). Knee flexion and extension strength and $\mathrm{H} / \mathrm{Q}$ ratio in high-level track and field athletes. Isokinetics and Exercise, 14(3): 279-289.

OSTERNIG, L.R. (1975). Optimal isokinetic loads and velocities producing muscular power in humans. Archives of Physical Medicine and Rehabilitation, 56: 152-155.

PERRIN, D.H. (1993). Isokinetic exercise and assessment. Champaign, IL: Human Kinetics.

TAYLOR, N.A.S.; SANDERS, H.A.; HOWICK, E.I. \& STANLEY, S.N. (1991). Static and dynamic assessment of the Biodex dynamometer. European Journal of Applied Physiology, 62(3): 180-188.

WILK, K.E.; ARRIGO, C.A. \& ANDREWS, J.R. (1993). Rehabilitation of the elbow in the throwing athlete. Journal of Orthopaedic and Sports Physical Therapy, 17: 305-317.

WRIGLEY, T. \& STRAUSS, G. (2000). Strength assessment by isokinetic dynamometry. In Australian Sports Commission (2000). Physiological tests for elite athletes. Champaign, IL: Human Kinetics.

Dr. Leon Lategan: School for Sport and Movement Sciences, University of Johannesburg, P.O. Box 524, Auckland Park, Johannesburg 2006, Republic of South Africa. Tel: +27 (0)11 489 2749, Fax: +27 (0)11 489 2671, E-mail: 1la@uj.ac.za 